\title{
Faraday effect in rippled graphene: Magneto-optics and random gauge fields
}

\author{
Jürgen Schiefele, ${ }^{1}$ Luis Martin-Moreno, ${ }^{2}$ and Francisco Guinea ${ }^{3,4}$ \\ ${ }^{1}$ Instituto de Ciencia de Materiales de Madrid, CSIC, E-28 049 Madrid, Spain \\ ${ }^{2}$ Instituto de Ciencia de Materiales de Aragón and Departamento de Física de la Materia Condensada, CSIC-Universidad de Zaragoza, \\ E-50 009 Zaragoza, Spain \\ ${ }^{3}$ IMDEA Nanociencia, Calle de Faraday 9, E-28 049 Madrid, Spain \\ ${ }^{4}$ Department of Physics and Astronomy, University of Manchester, Oxford Road, Manchester M13 9PL, United Kingdom
}

(Received 15 December 2015; revised manuscript received 11 June 2016; published 1 July 2016)

\begin{abstract}
A beam of linearly polarized light transmitted through magnetically biased graphene can have its axis of polarization rotated by several degrees after passing the graphene sheet. This large Faraday effect is due to the action of the magnetic field on graphene's charge carriers. As deformations of the graphene membrane result in pseudomagnetic fields acting on the charge carriers, the effect of random mesoscopic corrugations (ripples) can be described as the exposure of graphene to a random pseudomagnetic field. We aim to clarify the interplay of these typically sample inherent fields with the external magnetic bias field and the resulting effect on the Faraday rotation. In principle, random gauge disorder can be identified from a combination of Faraday angle and optical spectroscopy measurements.
\end{abstract}

DOI: 10.1103/PhysRevB.94.035401

\section{INTRODUCTION}

Elastic scattering of light by a conducting medium, and thus the reflection and absorption of a beam of light passing through a slab of material, are largely determined by the interaction of the beam with the material's free electrons. If an external magnetic field is applied to the medium, the Lorentz force acts on the conduction electrons, and if, for definiteness, the electric component of the incident beam is linearly polarized along the $x$ direction (see Fig. 1), the transmitted as well as the reflected beam both acquire a component along the $y$ direction. This rotation of the polarization direction in the reflected (transmitted) beam is called the Kerr (Faraday) effect. The direction of the rotation depends only on the direction of the magnetic field, not on the propagation direction of light, such that the Faraday and Kerr effects enable nonreciprocal optical devices [1-3] such as optical isolators, which allow light to pass in one direction while blocking propagation in the reverse direction.

For the particular case of a beam of light of frequency $\Omega$ passing through an atomically thin sheet of monolayer graphene in a constant magnetic field of magnitude $B$, one finds that the polarization is rotated by the Faraday angle [4],

$$
\Theta_{F} \simeq \frac{1}{2} \operatorname{Re} \sigma_{x y}(\Omega, B) .
$$

Under typical experimental conditions, monolayer graphene can rotate the polarization by several degrees [5], a large effect considering that Faraday isolators based on conventional magneto-optical materials require propagation distances of several millimeters [1]. Equation (1) neglects terms of higher than linear order in the fine-structure constant, and it is assumed that the magnetic field as well as the propagation direction of the incident light are both perpendicular to the graphene surface. $\sigma_{x y}(\Omega, B)$ denotes the off-diagonal element of graphene's conductivity tensor, such that a measurement of $\Theta_{F}$ represents an optical (that is, $\Omega \neq 0$ ) analogue to a dc measurement of the Hall current.

In the static limit, the dc Hall conductivity of graphene is known to show a steplike behavior as a function of the filling factor $n_{0}[6-8]$,

$$
\sigma_{x y}(\Omega=0)=\frac{e^{2}}{h} 2\left(1+2 n_{0}\right),
$$

where $n_{0}$ marks the last filled level right below the Fermi energy $E_{F}$. For low carrier densities and large magnetic fields, the Landau levels around $E_{F}$ are well separated, and correspondingly, a Faraday angle displaying quantized steps as in Eq. (2) has been observed at $\Omega=1 \mathrm{THz}$ for graphene samples with $E_{F}=60 \mathrm{meV}$ and $B$ up to $7 \mathrm{~T}$ [9].

However, the spacing of graphene's Landau levels $E_{n}$ is nonequidistant [6], with the energy difference between adjacent levels getting smaller for higher $n$. Correspondingly, for modest magnetic fields and with carrier densities in typical graphene-on-substrate samples being far from the neutrality point, the experimentally observed $\Theta_{F}(\Omega)$ [10] follows the classical off-diagonal Drude conductivity [11-13],

$$
\sigma_{x y}(\Omega)=-\operatorname{sgn}(e B) \frac{e^{2}}{2 h} \frac{g_{s} g_{v} \omega_{c} E_{F}}{\hbar\left[(\Omega+i / \tau)^{2}-\omega_{c}^{2}\right]},
$$

which does not resolve the quantized Landau levels. Equation (3) is parametrized in terms of a cyclotron frequency [14],

$$
\omega_{c}=\frac{|e B| v_{F}^{2}}{\left|E_{F}\right|}
$$

where $v_{F}$ denotes the Fermi velocity, $g_{s}=g_{v}=2$ are factors for the spin and valley degeneracy, and $\tau$ is a phenomenological scattering time usually employed as a fitting parameter [10].

Figure 2 shows $\Theta_{F}$ versus frequency calculated with Eq. (3) for typical values of $E_{F}$ and $B$, and assuming $\tau=1.5 \times 10^{-13}$ $\mathrm{s}$, the value corresponding to the transport scattering time $\tau_{\mathrm{tr}}=$ $\hbar \mu v_{F} / e$ for a graphene sample with dc mobility $\mu=10000$ $\mathrm{cm}^{2} \mathrm{~V}^{-1} \mathrm{~s}^{-1}$ [15]. The rotation angle is seen to be maximal at frequencies slightly below $\omega_{c}$. Assuming $\omega_{c} \tau \gg 1$, which allows for closed cyclotron orbits between collisions, Eq. (3) 


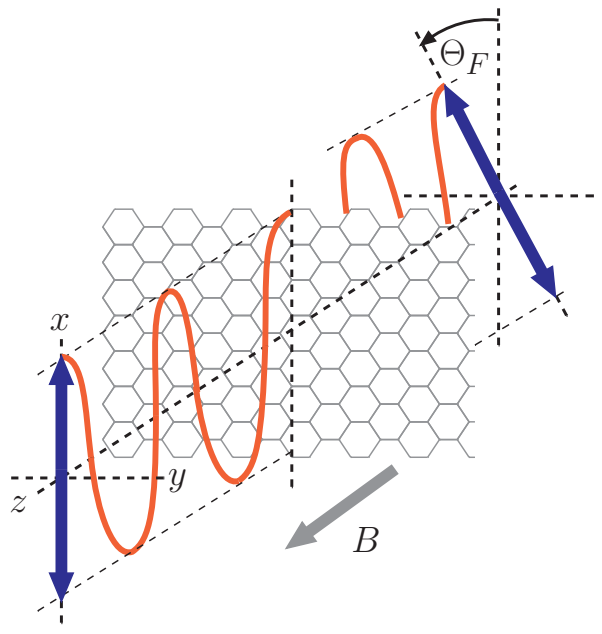

FIG. 1. The Faraday effect in graphene: The polarization plane of a linearly polarized incoming beam is rotated by the Faraday angle $\Theta_{F}$ after passing through graphene in a perpendicular magnetic field.

yields

$$
\Theta_{F}^{\max }=\frac{e^{2}}{h} \frac{E_{F}}{4 \hbar \omega_{c}}\left\{\omega_{c} \tau+1+O\left[1 /\left(\omega_{c} \tau\right)\right]\right\}
$$

for this maximal value of $\Theta_{F}$. From Eq. (5), it is clear that the magnitude of the achievable Faraday rotation strongly depends on $\tau$, that is, on the quality of the specific graphene sample.

Due to the particular structure of graphene's twodimensional crystal lattice, which results in a two-component spinor structure of the electron wave functions, it is not only the magnitude of disorder that determines carrier dynamics. Rather, a microscopic description of disorder effects in graphene has to distinguish between scalar and gauge potentials [6]. While the former are typically caused by charged impurities, the latter arise due to deformations of graphene's crystal lattice, and their effect on the charge carriers

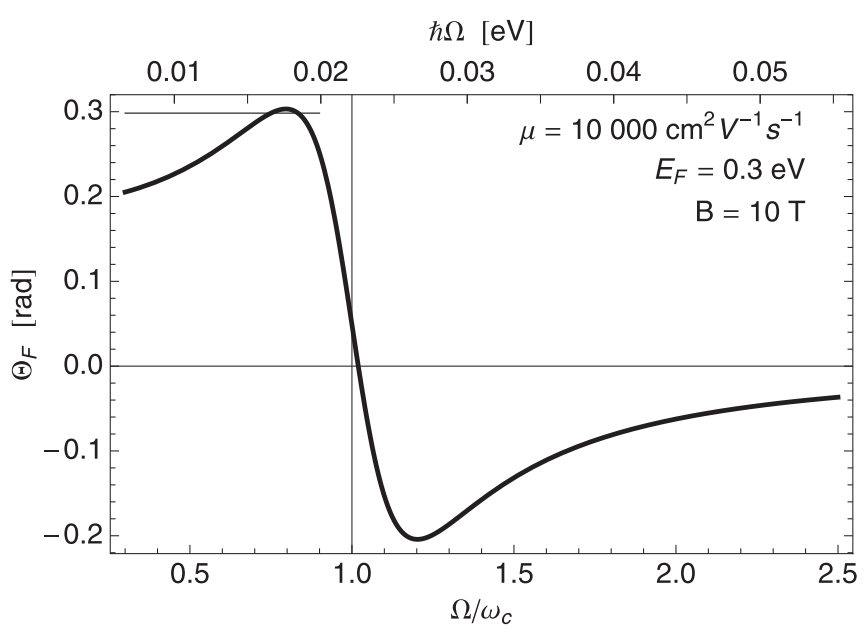

FIG. 2. Faraday rotation angle $\Theta_{F}$ vs frequency, calculated from the classical Drude formula (3) for $\sigma_{x y}$. The horizontal line indicates the maximal value of $\Theta_{F}$ [see Eq. (5)] at frequencies below the cyclotron frequency $\omega_{c}$. The scattering time has been set to $\tau=\tau_{\mathrm{tr}}=$ $1.5 \times 10^{-13} \mathrm{~s}$. can be described in terms of pseudomagnetic fields [16-18]. Pseudomagnetic fields affect carriers near the two inequivalent Fermi points $\mathbf{K}$ and $\mathbf{K}^{\prime}$ with an opposite sign, such that they either add up to or have to be subtracted from the (real) external bias field $B$ acting on the carriers [19,20]. As it has been found that the strain-induced pseudomagnetic field in graphene nanobubbles can lead to a Landau quantization of the charge carriers equivalent to field strengths of a few hundred Tesla [21], it seems possible to engineer the magneto-optical response of graphene via the controlled application of nonhomogeneous mechanical strain, if a constant pseudomagnetic field can be produced over a large spatial region [22,23].

Apart from such engineered strain distributions, random strain fields are present in most available graphene samples because graphene membranes, whether supported by a substrate material or in suspended devices, are never completely flat. Instead, they show a rippled structure [24], and for clean samples the pseudomagnetic fields caused by these mesoscopic corrugations have been shown to set the dominant limit on electronic transport [25]. Despite this importance of ripples for graphene's transport properties, experiments on the Faraday effect in graphene do not reveal any valley-dependent interplay between (potentially large) gauge fields and the magnetic bias field. Instead, the data is found to be fitted well by Eq. (3) with an appropriately chosen scattering time $[4,9,10]$.

It is the aim of the present work to investigate the effect of sample-inherent, random strain configurations on the magnetooptical properties of graphene, and to understand why rippleinduced effective magnetic fields of the form $B \pm \Delta B$ in each valley do not seem to play a role in graphene's Faraday effect. To this end, we compare the effect of random ripples on the Faraday rotation with that of random scalar scatterers.

\section{DISORDER SCATTERING IN GRAPHENE-GAUGE AND SCALAR POTENTIALS}

In order to relate the scattering parameter $\tau$ appearing in Eq. (3) to an underlying microscopic scattering mechanism, we assume random Gaussian disorder [26]. Denoting the Fourier transform of the scattering potential with $V_{\mathbf{q}}$, disorder scattering can be introduced in perturbation theory by the correlator $\left\langle V_{\mathbf{q}} \otimes V_{-\mathbf{q}}\right\rangle_{\text {dis }}$, where the average is taken over all possible disorder configurations. As the electron wave function $\Psi$ is a spinor of rank four in sublattice (superscripts $A$ and $B$ ) and valley space (subscripts $\mathbf{K}$ and $\mathbf{K}^{\prime}$ ) [27],

$$
\Psi=\left\{\psi_{\mathbf{K}}^{A}, \psi_{\mathbf{K}}^{B}, \psi_{\mathbf{K}^{\prime}}^{A}, \psi_{\mathbf{K}^{\prime}}^{B}\right\},
$$

the correlator forms a tensor,

$$
\left[\left\langle V_{\mathbf{q}} \otimes V_{-\mathbf{q}}\right\rangle_{\mathrm{dis}}\right]_{h i ; j k}, h, i, j, k \in\{1,2,3,4\} ;
$$

see Fig. 3(b).

We want to distinguish between a scalar and a gauge potential $V^{s, g}$, and consider

$$
\begin{aligned}
& \left\langle V_{\mathbf{q}}^{s} \otimes V_{-\mathbf{q}}^{s}\right\rangle_{\text {dis }}=g \mathbb{1} \otimes \mathbb{1}, \\
& \left\langle V_{\mathbf{q}}^{g} \otimes V_{-\mathbf{q}}^{g}\right\rangle_{\text {dis }}=g \gamma_{1} \otimes \gamma_{1},
\end{aligned}
$$

where $\mathbb{1}$ and $\gamma_{1}$ are $4 \times 4$ matrices. The scalar potential $V_{\mathbf{q}}^{s}$ affects carriers on the $A$ and $B$ sublattices of graphene and 
(a)

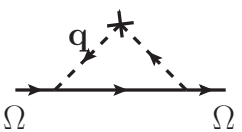

(c)

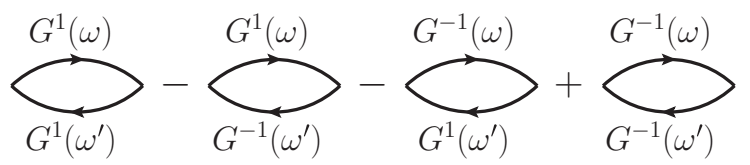

(d)

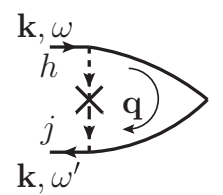

(e)

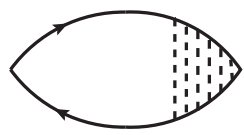

FIG. 3. Diagrammatic expressions appearing in the calculation of $\sigma_{x y}$. (a) Self-energy $\Sigma(\Omega)$; see Eq. (9). (b) Disorder correlator $\left\langle V_{\mathbf{q}} \otimes V_{-\mathbf{q}}\right\rangle_{\text {dis }}$; see Eq. (6). (c) Integral kernel of Eq. (13). (d) Vertex correction $\Gamma\left(\mathbf{k}, \omega, \omega^{\prime}\right)$; see Eq. (17). (e) Resummation of vertex corrections leading to Eq. (20).

in both $\mathbf{K}$ and $\mathbf{K}^{\prime}$ valleys in the same manner. In contrast, the gauge potential is proportional to $\gamma_{1}$, defined as

$$
\gamma_{i}=\left(\begin{array}{cc}
\sigma_{i} & 0 \\
0 & -\sigma_{i}
\end{array}\right),
$$

where we use the standard $2 \times 2$ Pauli matrices $\sigma_{i}$ acting on the sublattice index, that is, $A$ or $B$ site [6]. Thus, $V_{\mathbf{q}}^{g}$ affects carriers in each valley with opposite sign.

Apart from their different tensor structure, we have set both correlators proportional to a constant $g$ of dimensions $(\text { energy })^{2} \times$ area, as would be the case for sharply localized, $\delta$ like scattering centers. In a more detailed description, gauge as well as scalar disorder potentials are likely to show long-range correlations, resulting in an overall power-law dependence of the correlators in Eq. (7) [16,25]. As we will see below, the approximation with a momentum-independent constant $g$ greatly facilitates the evaluation of otherwise infrared-divergent loop integrals, while still taking into full account the scalar or gauge nature of the disorder. Further, the block-diagonal potentials in Eq. (7) neglect possible intervalley scattering processes, which have been shown to yield only weak contributions to the resistivity of graphene [25].

In order to assign a value to the disorder strength $g$, we aim to link it to the dc carrier mobility characteristic for each sample. We assume the dc conductivity of graphene to be of the Drude form, $\sigma_{x x}^{\mathrm{dc}}=\frac{e^{2}}{h} 2 v_{F} k_{F} \tau_{\mathrm{tr}}$, where $\tau_{\mathrm{tr}}$ is obtained from Fermi's rule together with a Boltzmann weighting factor $1-\cos \phi_{\mathbf{q}}$ which suppresses contributions of small-angle scattering events $\left(\cos \phi_{\mathbf{q}} \simeq 1\right)$ to the dc conductivity [15,25]. Then, the definition of $\mu$ as the electrical conductivity per carrier, $\mu=\sigma_{x x}^{\mathrm{dc}} /(n e)$, where $n$ is the carrier density, leads to

$$
\mu=\frac{e}{h} \frac{2 \pi v_{F} \tau_{\mathrm{tr}}}{k_{F}} .
$$

Transport measurements revealed that $\mu$ is independent of the carrier density [25], hence the scattering parameter $\tau_{\text {tr }}$ has to be proportional to $k_{F}$ and, as a function of $\mu$, has to fulfill the above relation.
The retarded electronic self-energy due to scattering at the potential $V^{s}$ or $V^{g}$ in Born approximation [see the diagram in Fig. 3(a)] is given by

$$
\begin{aligned}
\Sigma^{s, g}(\Omega)= & g\left(\hbar \Omega+E_{F}\right) \\
& \times \int_{0}^{\infty} \frac{q d q}{2 \pi} \frac{1}{\left(\hbar \Omega+E_{F}+i 0\right)^{2}-\left(\hbar v_{F} q\right)^{2}} .
\end{aligned}
$$

Importantly, it does not distinguish between a scalar or vector character of the underlying disorder. The imaginary part of $\Sigma(\Omega)$ describes the broadening of electronic momentum eigenstates due to the disorder potential, and defines a quantum scattering time $\tau_{q}$,

$$
\frac{1}{\tau_{q}}=-\left.\frac{2}{\hbar} \operatorname{Im} \Sigma\right|_{\Omega=0}=g \frac{k_{F}}{2 \hbar^{2} v_{F}} .
$$

For white-noise disorder, $\tau_{q}$ is known to be smaller than $\tau_{\text {tr }}$ by a factor of 2 [15], and taking Eq. (10) together with the requirement given by Eq. (8), we arrive at

$$
g=4 \pi \frac{e}{h \mu} \frac{\left(\hbar v_{F}\right)^{2}}{k_{F}^{2}}
$$

for the disorder strength in a graphene sample with mobility $\mu$.

\section{DISORDER CORRECTIONS TO THE HALL CONDUCTIVITY}

From Kubo's formula, the Hall conductivity of graphene can be expressed as $[28,29]$

$$
\begin{aligned}
\sigma_{x y}(\Omega)= & \frac{e^{2}}{h} \operatorname{Im} \frac{4 \pi v_{F}^{2}}{\Omega} \int d \omega d \omega^{\prime} \frac{n_{F}\left(\omega^{\prime}\right)-n_{F}(\omega)}{\omega-\omega^{\prime}-\Omega-i 0} \\
& \times \sum_{n=0}^{\infty} K_{n}^{(0)}\left(\omega, \omega^{\prime}\right),
\end{aligned}
$$

with $n_{F}$ the Fermi distribution, and the integral kernel $K_{n}^{(0)}$ resulting from a sum over bubble diagrams [see Fig. 3(c)],

$$
\begin{aligned}
& \sum_{n=0}^{\infty} K_{n}^{(0)}\left(\omega, \omega^{\prime}\right) \\
& \quad=\sum_{\beta, \gamma= \pm 1} \frac{\beta \gamma}{(2 \pi i)^{2}} \int \frac{d^{2} k}{(2 \pi)^{2}} \operatorname{Tr}\left[\gamma_{1} G^{\beta}(\omega, \mathbf{k}) \gamma_{2} G^{\gamma}\left(\omega^{\prime}, \mathbf{k}\right)\right] .
\end{aligned}
$$

The sum over the indices $\beta$ and $\gamma$ realizes different combinations of retarded and advanced Green's functions $G^{ \pm 1}$ for charge carriers, given in Eq. (A1).

Disorder corrections can enter Eq. (13) either through self-energy insertions [as shown in Fig. 3(a)], which shift the resonances of the Green's functions in the bubble diagrams of Fig. 3(c), or through vertex corrections, shown in Fig. 3(d); see Ref. [30].

\section{A. Self-energy insertions}

Within the lowest-order Born approximation, we assume the real part of disorder-induced self-energies to be negligible, 
such that they simply introduce the scattering time $\tau_{q}$ of Eq. (10) into the Green's functions in Eq. (13). For such a constant damping parameter, the bubble diagrams of Eq. (12) have been evaluated in Refs. [28,29], and we only recall the main steps of the calculation.

After performing the momentum integration in Eq. (13) with the help of relation (A3), $K_{n}^{(0)}$ can be expressed in terms of the polarization functions,

$$
\begin{aligned}
\Pi_{m n}^{\beta \gamma}\left(\omega, \omega^{\prime}\right)= & \frac{2\left(\hbar v_{F}\right)^{2}}{l_{B}^{2}} \frac{\hbar \omega+i \beta \hbar /\left(2 \tau_{q}\right)}{\hbar^{2}\left[\omega+i \beta /\left(2 \tau_{q}\right)\right]^{2}-E_{n}^{2}} \\
& \times \frac{\hbar \omega^{\prime}+i \gamma \hbar /\left(2 \tau_{q}\right)}{\hbar^{2}\left[\omega^{\prime}+i \gamma /\left(2 \tau_{q}\right)\right]^{2}-E_{m}^{2}},
\end{aligned}
$$

where the Landau levels are given by

$$
E_{n}=\operatorname{sign}(n) \hbar v_{F} \sqrt{2|n|} / l_{B},
$$

with $l_{B}=\sqrt{\hbar /|e B|}$ the magnetic length. We arrive at [29]

$$
\begin{aligned}
K_{n}^{(0)}\left(\omega, \omega^{\prime}\right)= & i \operatorname{sgn}(e B) \frac{g_{s} g_{v}}{4(2 \pi)^{3} v_{F}^{2}} \\
& \times \sum_{\beta, \gamma= \pm 1} \beta \gamma\left[\Pi_{n+1, n}^{\beta \gamma}\left(\omega, \omega^{\prime}\right)-\Pi_{n, n+1}^{\beta \gamma}\left(\omega, \omega^{\prime}\right)\right],
\end{aligned}
$$

with $g_{s}=g_{v}=2$ denoting spin and valley degeneracy, respectively.

\section{B. Vertex corrections}

Apart from self-energy corrections contributing to the scattering time, disorder introduces, in leading order of perturbation theory, a vertex correction,

$$
\begin{aligned}
& {\left[\Gamma^{\beta \gamma}\left(\mathbf{k}, \omega, \omega^{\prime}\right)\right]_{h j}} \\
& =\int \frac{d^{2} q}{(2 \pi)^{2}}\left[\left\langle V_{\mathbf{q}-\mathbf{k}} \otimes V_{-\mathbf{q}+\mathbf{k}}\right\rangle_{\mathrm{dis}}\right]_{h i ; j k} \\
& \quad \times\left[G^{\beta}(\omega, \mathbf{q}) \gamma_{2} G^{\gamma}\left(\omega^{\prime}, \mathbf{q}\right)\right]_{i k}
\end{aligned}
$$

[see Fig. 3(d)], which replaces the bare velocity operator $\gamma_{2}$ appearing on the right-hand side of Eq. (13). For $V_{\mathbf{q}}$, a constant independent of $\mathbf{q}$, the integration over the loop momentum in Eq. (17) can be performed using Eq. (A3), and for the scalar and gauge disorder of Eq. (7), we obtain

$$
\Gamma_{s, g}^{\beta \gamma}\left(\mathbf{k}, \omega, \omega^{\prime}\right)=\frac{g}{4 \pi\left(\hbar v_{F}\right)^{2}} \gamma_{2} \cdot \operatorname{diag}\left(a_{s, g}^{\beta \gamma}, b_{s, g}^{\beta \gamma}, a_{s, g}^{\beta \gamma}, b_{s, g}^{\beta \gamma}\right),
$$

where the entries $a$ and $b$ of the diagonal matrix are dimensionless functions of the frequencies $\omega$ and $\omega^{\prime}$,

$$
\begin{aligned}
& a_{s}^{\beta \gamma}\left(\omega, \omega^{\prime}\right)=b_{g}^{\beta \gamma}\left(\omega, \omega^{\prime}\right)=\sum_{\bar{n}=0}^{\infty} \Pi_{\bar{n}, \bar{n}+1}^{\beta \gamma}\left(\omega, \omega^{\prime}\right), \\
& b_{s}^{\beta \gamma}\left(\omega, \omega^{\prime}\right)=a_{g}^{\beta \gamma}\left(\omega, \omega^{\prime}\right)=\sum_{\bar{n}=0}^{\infty} \Pi_{\bar{n}+1, \bar{n}}^{\beta \gamma}\left(\omega, \omega^{\prime}\right),
\end{aligned}
$$

such that $\Gamma_{g}=\gamma_{1} \Gamma_{s} \gamma_{1}$.

Resumming the vertex correction (18) in a geometric series as indicated in Fig. 3(e) yields, for either scalar (superscript $s$ )

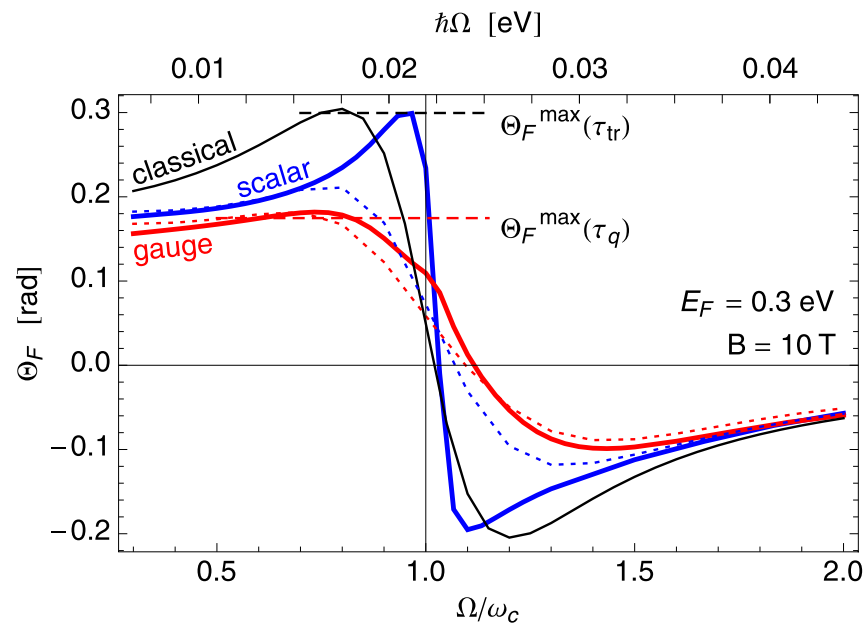

FIG. 4. Faraday rotation angle $\Theta_{F}$ calculated from the Kubo formula given by Eq. (20), for a graphene sample with $\mu=10000$ $\mathrm{cm}^{2} \mathrm{~V}^{-1} \mathrm{~s}^{-1}$ due to scalar (blue curve) and gauge disorder (red curve), respectively. For comparison, the black curve redisplays the classical model shown in Fig. 2. The scattering time $\tau_{q}$ in Eq. (14) has been set to $7.5 \times 10^{-14} \mathrm{~s}$, resulting in a disorder strength $g=0.058 \hbar v_{F}$. Horizontal dashed lines indicate the maximum angle given by Eq. (5). The dotted curves show the results obtained by using only terms up to linear order in $g$ in the integral kernel (22) of the Kubo formula.

or gauge (superscript $g$ ) disorder,

$$
\begin{aligned}
\sigma_{x y}^{s, g}(\Omega) & =\frac{e^{2}}{h} \operatorname{Im} \frac{2\left(\hbar v_{F}\right)^{2}}{\pi \Omega} \int d \omega d \omega^{\prime} \frac{n_{F}\left(\omega^{\prime}\right)-n_{F}(\omega)}{\omega-\omega^{\prime}-\Omega-i 0} \\
& \times i \operatorname{sign}(e B) \sum_{\gamma, \beta= \pm 1} K_{s, g}^{\beta \gamma}\left(\omega, \omega^{\prime}\right) .
\end{aligned}
$$

Here,

$$
\begin{aligned}
K_{s}^{\beta \gamma}\left(\omega, \omega^{\prime}\right)= & \frac{b_{s}^{\beta \gamma}\left(\omega, \omega^{\prime}\right)}{4 \pi\left(\hbar v_{F}\right)^{2}-g b_{s}^{\beta \gamma}\left(\omega, \omega^{\prime}\right)} \\
& -\frac{a_{s}^{\beta \gamma}\left(\omega, \omega^{\prime}\right)}{4 \pi\left(\hbar v_{F}\right)^{2}-g a_{s}^{\beta \gamma}\left(\omega, \omega^{\prime}\right)}, \\
K_{g}^{\beta \gamma}\left(\omega, \omega^{\prime}\right)= & \frac{a_{g}^{\beta \gamma}\left(\omega, \omega^{\prime}\right)}{4 \pi\left(\hbar v_{F}\right)^{2}-g b_{g}^{\beta \gamma}\left(\omega, \omega^{\prime}\right)} \\
& -\frac{b_{g}^{\beta \gamma}\left(\omega, \omega^{\prime}\right)}{4 \pi\left(\hbar v_{F}\right)^{2}-g a_{g}^{\beta \gamma}\left(\omega, \omega^{\prime}\right)} .
\end{aligned}
$$

As the optical response of doped graphene is due to excitation of carriers close to the Fermi level, the sum over Landau levels in (19) is dominated by only a few terms around the filling factor, $n_{0}=\left\lfloor E_{F}^{2} / E_{1}^{2}\right\rfloor$. For our parameters, the main contributions are found to come from the level $n=n_{0}$ and $n=n_{0}+1$, and we only retain these two terms in the numerical evaluation of Eq. (20).

Within this approximation, the Faraday angle calculated from $\sigma_{x y}^{s}$ and $\sigma_{x y}^{g}$ is shown in Fig. 4, where the blue curve corresponds to scalar disorder and the red one to gauge disorder. Assuming a graphene sample with $\mu=10000$ $\mathrm{cm}^{2} \mathrm{~V}^{-1} \mathrm{~s}^{-1}$, we set $\tau_{q}=\tau_{\text {tr }} / 2=7.5 \times 10^{-14}$. Comparing with the classical expression (3) shown in Fig. 2, we note 
that neither type of disorder changes the functional form of $\Theta_{F}(\Omega)$ : The frequency of maximal rotation remains slightly below $\omega_{c}$ of Eq. (4), and hence is determined by the external magnetic field. This is in good accordance with experimental observations, where the data can be captured well by Eq. (3), if $\tau$ is treated as a fitting parameter. In contrast, a naive model of gauge disorder introducing a constant effective field $B_{\text {eff }}=B \pm \Delta B$ for carriers in the $\mathbf{K}$ and $\mathbf{K}^{\prime}$ valley, respectively, would shift the effective resonance frequencies in Eq. (3) to $\omega_{c}^{ \pm}=v_{F}^{2}|e(B \pm \Delta B)| /\left|E_{F}\right|$.

Concerning the magnitude of the achievable rotation angle, Fig. 4 shows a larger value for scalar disorder compared to gauge disorder, which stems from the different contribution of the vertex corrections to $\sigma_{x y}^{s}$ and $\sigma_{x y}^{g}$. In particular, upon expanding (21) as

$$
\begin{aligned}
K_{s}^{\beta \gamma}\left(\omega, \omega^{\prime}\right)= & \frac{1}{4 \pi\left(\hbar v_{F}\right)^{2}}\left(b_{s}^{\beta \gamma}-a_{s}^{\beta \gamma}\right) \\
& +\frac{g}{8 \pi^{2}\left(\hbar v_{F}\right)^{4}}\left[\left(b_{s}^{\beta \gamma}\right)^{2}-\left(a_{s}^{\beta \gamma}\right)^{2}\right]+O\left(g^{2}\right) \\
K_{g}^{\beta \gamma}\left(\omega, \omega^{\prime}\right)= & \frac{1}{4 \pi\left(\hbar v_{F}\right)^{2}}\left(b_{s}^{\beta \gamma}-a_{s}^{\beta \gamma}\right)+O\left(g^{2}\right)
\end{aligned}
$$

we note that in the case of gauge disorder, vertex corrections to the simple bubble diagrams are absent at linear order in $g$. The simple bubble diagrams given by the $O\left(g^{0}\right)$ terms of Eq. (22) are known to reproduce the classical Drude formula of Eq. (3), with $\tau=\tau_{q}$ (see Appendix B for details.) Consequently, Fig. 4 shows the maximal rotation angle in a sample with gauge disorder to be well approximated by $\Theta_{F}^{\max }\left(\tau_{q}\right)$, whereas the classical $\Theta_{F}^{\max }\left(\tau_{\mathrm{tr}}\right)$ is restored by the contribution of vertex corrections for scalar disorder [31].

\section{CONCLUSIONS}

The dynamics of charge carriers which simultaneously experience the presence of a pseudomagnetic field $\Delta B$ as well as an external bias field $B$ is governed by an overall effective field $B \pm \Delta B$, depending on whether the carriers reside near the $\mathbf{K}$ or $\mathbf{K}^{\prime}$ point in graphene's Brillouin zone. This results in a different spectrum of Landau levels for $\mathbf{K}$ and $\mathbf{K}^{\prime}$ carriers $[19,20]$, and, in terms of the classical Hall conductivity $\sigma_{x y}$ of Eq. (3), one might therefore expect carriers at $\mathbf{K}$ and $\mathbf{K}^{\prime}$ to contribute to the Faraday effect with an effective cyclotron frequency of $\omega_{c}^{ \pm}$, respectively. However, instead of resulting in an approximately constant pseudomagnetic field over an extended area, the effect of sample-inherent ripples rather corresponds to the exposure of graphene to a random magnetic field, varying in direction and magnitude on a length scale of $\simeq 100 \AA$ [24].

The above calculation of $\sigma_{x y}$, which regards the random pseudomagnetic field as a sublattice-mixing disorder potential, predicts that the frequency dependence of $\Theta_{F}$ is similar for both scalar- and gauge-disorder mechanisms. Consequently, experimental data on Faraday rotation can be fitted well by assuming either scalar or gauge disorder, if an appropriate $\tau$ is used as a phenomenological fitting parameter. However, we suggest that in case the quantum scattering time $\tau_{q}$ of the specific sample is known, the magnitude of the
Faraday rotation might allow one to differentiate between samples with predominantly gauge disorder and those showing predominantly scalar disorder. An experimental possibility would be to determine $\tau_{q}$ by spectroscopic observation of the width of the relevant Landau levels near the Fermi energy [32], and compare it with the scattering time extracted from the maximum Faraday rotation observed in the same sample.

While our calculations do not predict a shift of the observable effective $\omega_{c}$ due to random strain fields, we wish to emphasize that this does not exclude the possibility to influence the magneto-optical properties of graphene with appropriately engineered macroscopic strain configurations.

\section{ACKNOWLEDGMENTS}

The research leading to these results has received funding from the European Union Seventh Framework Programme under Grant Agreement No. 604391 Graphene Flagship, from the European Research Council through Grant No. 290846, and from the Ministerio de Economía y Competitividad (MINECO), Spain under Projects No. MAT2014-53432-C5$1-\mathrm{R}$ and No. FIS2014-207432. The authors are grateful for the hospitality of the Donostia International Physics Center (DIPC, San Sebastian), where part of this work was concluded.

\section{APPENDIX A: ELECTRON PROPAGATOR AND WAVE FUNCTIONS}

The retarded (advanced) propagator for two-dimensional charge carriers in graphene reads $[29,33]$

$$
G^{\beta}(\omega, \mathbf{k}, \tau)=e^{-\mathbf{k}^{2} l_{B}^{2}} \sum_{n=0}^{\infty} \frac{(-1)^{n} G_{n}^{\beta}(\omega, \mathbf{k}, \tau)}{[\hbar \omega+i \beta \hbar /(2 \tau)]^{2}-E_{n}^{2}},
$$

where $\beta= \pm 1$ and $E_{n}$ denotes the energy of the $n$th Landau level; see Eq. (15). The numerator of Eq. (A1) is given by

$$
\begin{aligned}
G_{n}^{\beta}(\omega, \mathbf{k}, \tau)= & 2[\hbar \omega+i \beta \hbar /(2 \tau)] \gamma^{0} \\
& \times\left\{L_{n}^{(0)}\left(2 \mathbf{k}^{2} l_{B}^{2}\right)\left[1-i \gamma^{1} \gamma^{2} \operatorname{sign}(e B)\right] / 2\right. \\
& -\left(1-\delta_{n, 0}\right) L_{n-1}^{(0)}\left(2 \mathbf{k}^{2} l_{B}^{2}\right) \\
& \left.\times\left[1+i \gamma^{1} \gamma^{2} \operatorname{sign}(e B)\right] / 2\right\} \\
& -\left(1-\delta_{n, 0}\right) 4 \hbar v_{F} \mathbf{k} \cdot \vec{\gamma} L_{n-1}^{(1)}\left(2 \mathbf{k}^{2} l_{B}^{2}\right) .
\end{aligned}
$$

The associated Laguerre polynomials $L_{n}^{(\alpha)}(x)$ appearing in Eq. (A2) fulfill the orthogonality relation [34]

$$
\int_{0}^{\infty} d x x^{\alpha} e^{-x} L_{n}^{(\alpha)}(x) L_{m}^{(\alpha)}(x)=\frac{\Gamma(n+\alpha+1)}{n !} \delta_{n, m} .
$$

Together with the identity

$$
L_{n}^{(m-n)}(x)=\frac{m !}{n !}(-x)^{n-m} L_{m}^{(n-m)}(x)
$$

(see Ref. [34]), Eq. (A3) allows one to compute the momentum integrals appearing in Eqs. (13) and (17) of the main text.

The presence of Laguerre polynomials in Eq. (A2) results from overlap integrals of Hermite polynomials $H_{n}(x)$,

$$
\begin{aligned}
& \int d x e^{-x^{2}} H_{m}(x+y) H_{n}(x+z) \\
& =\sqrt{\pi} 2^{n} m ! z^{n-m} L_{m}^{(n-m)}(-2 y z) \text { for } m \leqslant n .
\end{aligned}
$$


The Hermite polynomials, in turn, appear in the electron wave functions in the presence of a magnetic field; see Ref. [35].

\section{APPENDIX B: EVALUATING BUBBLE DIAGRAMS}

The simple bubble diagrams treated in Sec. III A allow, apart from numerical evaluation of Eq. (12) with the kernel $K_{n}^{(0)}$ of Eq. (16), a further analytical treatment: One of the frequency integrations in Eq. (12) can be performed noting that by construction, $K_{n}^{(0)}$ is a purely imaginary function of $\omega$ and $\omega^{\prime}$, and fulfills $K_{n}^{(0)}\left(\omega, \omega^{\prime}\right)=-K_{n}^{(0)}\left(\omega^{\prime}, \omega\right)$, such that we can rewrite (12) as

$$
\sigma_{x y}(\Omega)=\frac{e^{2}}{h} \operatorname{Im} \frac{4 \pi v_{F}^{2}}{\Omega} \sum_{n=0}^{\infty} \int d \omega n_{F}(\omega) \mathcal{K}_{n}^{(0)}(\Omega, \omega),
$$

where

$$
\mathcal{K}_{n}^{(0)}(\Omega, \omega)=\int \frac{d \omega^{\prime} K_{n}^{(0)}\left(\omega, \omega^{\prime}\right)}{\omega-\omega^{\prime}+\Omega+i 0}-\int \frac{d \omega^{\prime} K_{n}^{(0)}\left(\omega, \omega^{\prime}\right)}{\omega-\omega^{\prime}-\Omega-i 0} .
$$

The $d \omega^{\prime}$ integration in Eq. (B2) can be solved by closing the contour in the complex $\omega^{\prime}$ plane; the integrals that appear are of the form

$$
\begin{aligned}
& \int \frac{d \omega^{\prime}}{2 \pi i} \frac{\omega^{\prime} \pm i /(2 \tau)}{\left[\omega-\omega^{\prime} \pm(\Omega+i 0)\right]\left\{\left[\omega^{\prime} \pm i /(2 \tau)\right]^{2}-E_{n}^{2}\right\}} \\
& =\frac{\Omega \pm \omega+i /(2 \tau)}{E_{n}^{2}-[\Omega \pm \omega+i /(2 \tau)]^{2}} .
\end{aligned}
$$

With $\mathcal{K}_{n}^{(0)}$ given by an analytical expression, $\sigma_{x y}$ of Eq. (B1) can then be evaluated by numerically performing the remaining frequency integration and truncating the sum over Landau levels at a suitable cutoff value.

In the classical regime, the spacing between single Landau levels at the Fermi energy is much smaller than the Fermi energy itself, and $\left(E_{n_{0}+1}-E_{n_{0}}\right)^{2} \approx\left(\hbar \omega_{c}\right)^{2}$. In this limit, one can proceed to approximate the integrand in Eq. (B1) and perform the remaining frequency integration. This yields the classical Drude formula of Eq. (3), with $\tau=\tau_{q}$, plus an additional interband term which is nonresonant for $\Omega \ll 2 E_{F}$. For a detailed presentation of the calculational steps, we refer the reader to Ref. [28].
[1] J. Y. Chin, T. Steinle, T. Wehlus, D. Dregely, T. Weiss, V. I. Belotelov, B. Stritzker, and H. Giessen, Nat. Commun. 4, 1599 (2013).

[2] B. Peng, S. K. Özdemir, F. Lei, F. Monifi, M. Gianfreda, G. L. Long, S. Fan, F. Nori, C. M. Bender, and L. Yang, Nat. Phys. 10, 394 (2014).

[3] L. Bi, J. Hu, P. Jiang, D. H. Kim, G. F. Dionne, L. C. Kimerling, and C. A. Ross, Nat. Photon. 5, 758 (2011).

[4] I. Fialkovsky and D. Vassilevich, Eur. Phys. J. B 85, 1 (2012).

[5] We use units $\epsilon_{0}=c=1$, such that $e^{2} / h=2 \alpha=\frac{2}{137}$ radians. In the typical experimental setting (see, for example, Ref. [10]), graphene is deposited on a dielectric substrate. Depending on the refractive index of the specific substrate material used, the prefactor $\frac{1}{2}$ in Eq. (1) is modified, such that the rotation angles reached with graphene-on-SiC devices are by a factor of $\simeq 1.7$ smaller than those we show for freestanding graphene in Fig. 2.

[6] A. H. Castro Neto, F. Guinea, N. M. R. Peres, K. S. Novoselov, and A. K. Geim, Rev. Mod. Phys. 81, 109 (2009).

[7] K. S. Novoselov, A. K. Geim, S. V. Morozov, D. Jiang, M. I. Katsnelson, I. V. Grigorieva, S. V. Dubonos, and A. A. Firsov, Nature (London) 438, 197 (2005).

[8] Y.Zhang, Y.-W. Tan, H. L. Stormer, and P. Kim, Nature (London) 438, 201 (2005).

[9] R. Shimano, G. Yumoto, J. Y. Yoo, R. Matsunaga, S. Tanabe, H. Hibino, T. Morimoto, and H. Aoki, Nat. Commun. 4, 1841 (2013).

[10] I. Crassee, J. Levallois, A. L. Walter, M. Ostler, A. Bostwick, E. Rotenberg, T. Seyller, D. van der Marel, and A. B. Kuzmenko, Nat. Phys. 7, 48 (2011).

[11] N. W. Ashcroft and N. D. Mermin, Solid State Physics, 1st ed. (Brooks Cole, Belmont, CA, 1976)

[12] D. L. Sounas and C. Caloz, Appl. Phys. Lett. 98, 021911 (2011).

[13] M. Tymchenko, A. Y. Nikitin, and L. Martín-Moreno, ACS Nano 7, 9780 (2013).
[14] If we assume that the Lorentz force results in a circular electronic motion with angular frequency $\omega_{c}$, then $m^{*} \ddot{\mathbf{r}}=-e \dot{\mathbf{r}} \times \mathbf{B} / c$ with the effective electron mass $m^{*}=\left|E_{F}\right| / v_{F}^{2}$ (see Ref. [6]) leads to Eq. (4).

[15] E. H. Hwang and S. Das Sarma, Phys. Rev. B 77, 195412 (2008).

[16] F. Guinea, B. Horovitz, and P. Le Doussal, Phys. Rev. B 77, 205421 (2008).

[17] M. Vozmediano, M. Katsnelson, and F. Guinea, Phys. Rep. 496, 109 (2010).

[18] B. Amorim, A. Cortijo, F. de Juan, A. Grushin, F. Guinea, A. Gutiérrez-Rubio, H. Ochoa, V. Parente, R. Roldán, P. San-Jose, J. Schiefele, M. Sturla, and M. Vozmediano, Phys. Rep. 617, 1 (2016).

[19] B. Roy, Z.-X. Hu, and K. Yang, Phys. Rev. B 87, 121408 (2013).

[20] T. Low and F. Guinea, Nano Lett. 10, 3551 (2010).

[21] N. Levy, S. A. Burke, K. L. Meaker, M. Panlasigui, A. Zettl, F. Guinea, A. H. C. Neto, and M. F. Crommie, Science 329, 544 (2010).

[22] F. Guinea, M. I. Katsnelson, and A. K. Geim, Nat. Phys. 6, 30 (2010).

[23] S. Zhu, J. A. Stroscio, and T. Li, Phys. Rev. Lett. 115, 245501 (2015).

[24] J. C. Meyer, A. K. Geim, M. I. Katsnelson, K. S. Novoselov, T. J. Booth, and S. Roth, Nature (London) 446, 60 (2007).

[25] N. J. G. Couto, D. Costanzo, S. Engels, D.-K. Ki, K. Watanabe, T. Taniguchi, C. Stampfer, F. Guinea, and A. F. Morpurgo, Phys. Rev. X 4, 041019 (2014).

[26] E. Akkermans and G. Montambaux, Mesoscopic Physics of Electrons and Photons, 1st ed. (Cambridge University Press, Cambridge, 2007).

[27] See Ref. [6]. Here we neglect the electronic spin degree of freedom.

[28] V. P. Gusynin, S. G. Sharapov, and J. P. Carbotte, J. Phys. Condens. Matter 19, 026222 (2007). 
[29] V. P. Gusynin and S. G. Sharapov, Phys. Rev. B 73, 245411 (2006).

[30] U. Briskot, I. A. Dmitriev, and A. D. Mirlin, Phys. Rev. B 87, 195432 (2013).

[31] Reference [30] shows, for the case of $\sigma_{x x}$ in the presence of scalar disorder, how a resummation of self-energy insertions and vertex corrections approximately restores the appearance of the transport scattering time $\tau_{\text {tr }}$ in Eq. (3).
[32] P. Neugebauer, M. Orlita, C. Faugeras, A.-L. Barra, and M. Potemski, Phys. Rev. Lett. 103, 136403 (2009).

[33] V. A. Miransky and I. A. Shovkovy, Phys. Rep. 576, 1 (2015).

[34] I. Gradshteyn and I. Ryzhik, Table of Integrals, Series and Products, 6th ed., edited by A. Jeffrey and D. Zwillinger (Academic, New York, 2000).

[35] Y. Zheng and T. Ando, Phys. Rev. B 65, 245420 (2002). 